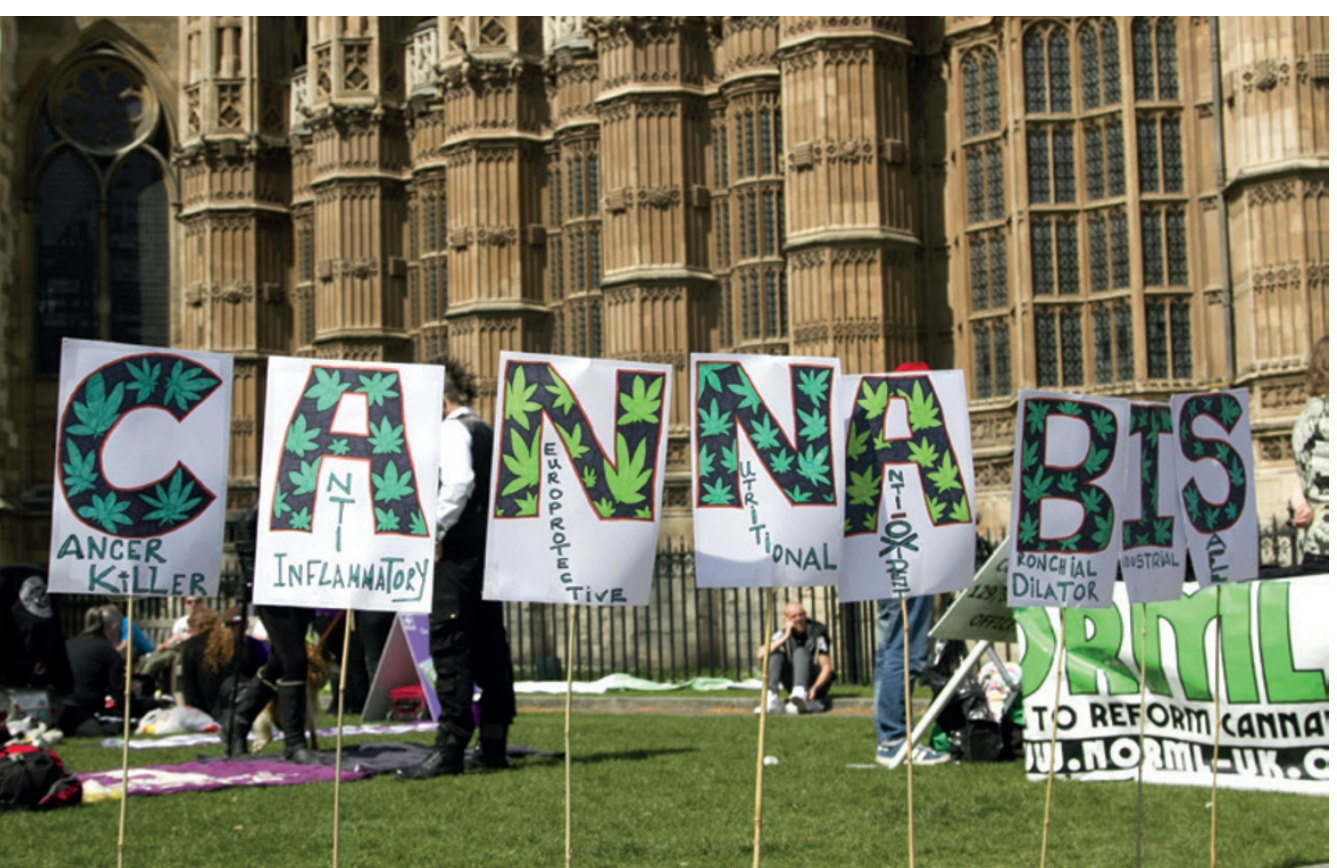

Proponents' claims about the medical benefits of marijuana are outpacing the evidence.

MEDICAL MARIJUANA

\title{
Showdown at the cannabis corral
}

\section{Researchers are gathering clinical data for medical marijuana against abackdrop of deregulation and opportunism.}

\section{BY MICHAEL EISENSTEIN}

Mary Jane Rathbun was beloved among patients with AIDS at San Francisco General Hospital in the late 1980s and early 1990s. As a volunteer, she took them for X-rays, filed their prescriptions - and supplied them with marijuana-laced brownies to alleviate their debilitating pain and wasting symptoms.

That service resulted in 'Brownie Mary' being arrested on multiple occasions. But patients got relief from her deliveries, and doctors both at her hospital and elsewhere were taking note. Barth Wilsey, then a pain research fellow at the University of California, San Francisco, also heard patients claiming they benefitted from this illicit substance. "I had a number of patients going to a dispensary in Oakland who told me that they were getting more relief from marijuana than from the medicine I gave them," he says. Inspired by Brownie Mary, Donald Abrams, an oncologist at the same hospital, tried mounting a clinical trial for medical marijuana as a treatment for patients with AIDS, but his efforts yielded years of frustration. He recalls a 1997 conversation with Alan Leshner, then head of US drug research agency NIDA. "He told me that they're the National Institute 'on' Drug Abuse, not 'for' Drug Abuse," says Abrams.

Much has changed since then. Abrams, Wilsey and others have steadily accumulated data that suggest that medical marijuana has a clinical benefit for treating chronic pain, and the barriers that previously thwarted research are eroding. In June, the US government lifted a major hurdle in the grant-review process for cannabis research by removing the requirement for Public Health Service (PHS) review, and NIDA is beginning to offer researchers a broader range of cannabis strains. Meanwhile, several countries and nearly half of all US states authorize medicinal marijuana use. These changes create opportunities for researchers to embark on more robust trials and to directly observe whether the herb helps patients.

However, medical marijuana may also be a victim of its own success. Marijuana laws make assumptions about medical benefits that outpace the evidence, and in some cases create markets for retailers to peddle unproven medicines - touting, for example, cannabis-derived oils to 'cure your own cancer'. Arno Hazekamp, who heads research and education at Bedrocan in Veendam, the Netherlands - the Dutch government's official provider of medical cannabis for more than a decade - dubs such opportunists "cannabis cowboys". "Everybody is selling stuff, but the real professionals who are sup posed to do the job are not there yet," he says. As a result, some of cannabis's scientific supporters fear that poorly planned deregulation could undermine efforts to establish medical legitimacy for this controversial crop.

\section{THE PROMISE OF POT}

Much of the seminal clinical research into medical marijuana (that is, in its herbal form) was performed under the auspices of the Center for Medical Cannabis Research (CMCR), funded by the state of California between 2000 and 2003. With CMCR support, Abrams and colleagues conducted a randomized trial showing that $52 \%$ of cannabis users reported a meaningful reduction in HIV-associated neuropathic pain compared with $24 \%$ of the control group ${ }^{1}$. With the help of a funding initiative from Health Canada, pain specialist Mark Ware and his team at McGill University in Montreal reported similar findings in patients with neuropathic pain as a result of a range of conditions ${ }^{2}$.

Early data for several conditions are intriguing, but limited. After learning that her patients with Crohn's disease were finding relief with cannabis, gastroenterologist Timna Naftali at Israel's Meir Medical Center in Kfar Saba and her team investigated whether medical marijuana can induce remission of the disease. They found that it significantly improved appetite and pain symptoms, and allowed some patients to end their dependency on steroids. However, cannabis had no clear effect on the inflammatory processes that underlie Crohn's disease, and did not result in remission" " The question is whether they were feeling better because it is a painkiller or reduces stress, or whether it really does something to inflammation," says Naftali.

Other findings are more tentative or anecdotal. Parents of children with severe epilepsy have reported dramatic improvements from cannabis oils derived from strains rich in cannabidiol $^{4}(\mathrm{CBD})$ - one of the plant's non-psychoactive chemicals. Studies of marijuana for the treatment of mental health conditions such as post-traumatic stress disorder (PTSD) have yielded ambiguous results: some suggesting that individuals with PTSD may self-medicate to achieve restful sleep - whereas other data suggest that marijuana can actually disrupt healthy sleep patterns. "The PTSD literature is a mess," says Mitchell Earleywine, a psychologist at the University of Albany, State University of New York. He queries whether the different results arise from patients using different 

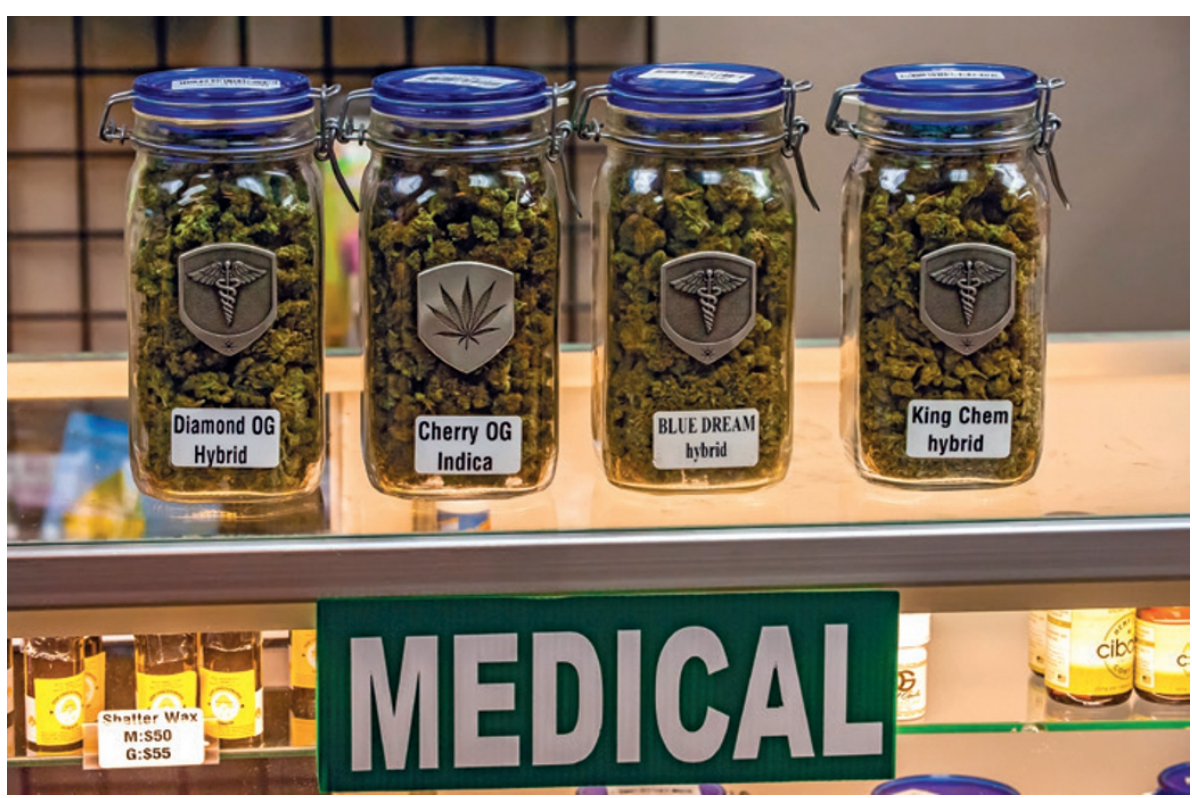

Medical marijuana is sold in dispensaries, but some people fear that it will be used recreationally.

marijuana strains, but is also concerned that the herb may offer limited long-term value to these patients by acting as a "Band-Aid" for a condition that could benefit more from direct psychiatric intervention. "Personally, I'd rather see these folks do behavioural treatments."

\section{QUALITY CONTROL}

The empirical basis for medical marijuana is thin. A systematic review of clinical studies published in June found "low-quality evidence" for the use of cannabis in almost all conditions, with the exception of chronic pain and muscle spasticity in multiple sclerosis $^{5}$. The trials were often confounded by poor design or execution, or failed to objectively demonstrate clinical benefit. Most studies examined isolated cannabinoids, either natural or synthetic; data from trials in which the plant is smoked or vaporized were especially limited.

Even the best trials were narrow in duration and scope. "These studies do not extend beyond more than two to four weeks, and are limited in the range of doses that are available in terms of THC [tetrahydrocannabinol] potency," says Ware. Finding a placebo for a trial of a drug with well-known psychoactive properties can be problematic. Margaret Haney, a neurobiologist at Columbia University in New York, notes that there is a considerable expectancy effect - preconceptions about cannabis skew how users respond. "It gets you high, and if you believe it's going to cure everything under the sun, then when you smoke it you feel like it cures everything under the sun," she says. And for conditions such as pain or appetite control that rely on user reports, expectations will have a big impact. Possible solutions include cannabis strains that are devoid of psychoactive THC, or placebos laced with mild sedatives.

The barriers facing even well-designed trials can be exceedingly high. In the United States, cannabis is a schedule 1 controlled substance (it has no currently accepted medical use). Studying the drug requires researchers to grapple with an alphabet soup of agencies, including NIDA, the Food and Drug Administration (FDA), the Drug Enforcement Agency (DEA) and until recently the PHS. The cost of largescale, long-term human studies can also be prohibitive. These bureaucratic and financial barriers have led to something of a stalemate, where researchers have no robust clinical data, but also lack the wherewithal to produce any. "If we just had a large funding source for even one good randomized controlled trial on a disorder that people care about, that would do the trick," says Earleywine.

US researchers are now seeing some hope. The lifting of the PHS review requirement should accelerate grant review. "The first time I studied marijuana, that stage took 18 months, and I couldn't go to the DEA or FDA until they concluded their review," says Wilsey. And NIDA's expansion of the range of strains available through its one authorized growing facility (at the University of Mississippi) means that researchers will have access to plants with THC levels that more closely resemble those found in street or dispensary cannabis as well as strains with higher levels of CBD.

However, the rapid policy changes now underway in North America will not necessarily help efforts to evaluate cannabis's medicinal value. Canada first established a legal medical cannabis framework in 2001, but the initial system proved impractical. "Health Canada put this very complicated programme into place, and only a few hundred people went through the process and got approved," says Benedikt Fischer, a public health researcher at the Centre for Addiction and Mental Health in Toronto. A series of court decisions led to the launch of a reformed programme in April 2014; now the government oversees cannabis production but not prescription access, which is at the discretion of individual doctors. "It's completely wide open - in many ways, arbitrary," says Fischer.

If Canada's system is arbitrary, the situation in the United States could be described as bewildering. At one extreme are states such as California, where patients can obtain almost any strain of cannabis by finding a doctor willing to provide a recommendation - merely a formality in some locales. By contrast, Minnesota offers restricted access to limited quantities of cannabis extract in liquid or pill form through state-run dispensaries for a narrow range of disorders. So far, 23 states and the District of Columbia have each come up with distinct approaches for providing medical cannabis - all in open defiance of federal law (see page S2). "There is no standardization," says J. Michael Bostwick, a psychiatrist at the Mayo Clinic in Rochester, Minnesota. "I guess we could have 'best practices' for states defying federal law, but that seems like adding convoluted craziness to what is already a crazy situation."

North of the border, the Canadian Medical Association and other professional organizations oppose the medical marijuana policy because they say it is based on insufficient evidence. Pharmacologist Harold Kalant of the University of Toronto notes that many doctors resent being appointed gatekeepers for this unproven drug - but even as a critic of the Canadian system, he believes the medical community has a responsibility to engage with this issue. "Who is better equipped to do this?" asks Kalant. "But this means they have to buckle down and do some reading and make themselves knowledgeable." And that includes keeping abreast of the latest thinking about potential risks (see 'Calculating the costs').

The problems associ"Those who ated with implementing areveryill can a medical marijuana law get cannabis are in part attributable get cannabis to the fact that this decianyway, and sion, which requires don't need a $\quad \begin{aligned} & \text { sion, which requires } \\ & \text { sophisticated scientific }\end{aligned}$ study." understanding, has been given to the public. "If the majority of voters are in favour of overall legalization, then let's just make it consistent across states," says Haney, "but don't have people vote on whether it's a medicine." Among jurisdictions that limit medical marijuana to specific conditions, there is little consensus on the best approach, whereas those that have adopted more open-ended policies have created a milieu of easily-obtained permits and abundant dispensaries that are largely indistinguishable from outright legalization. "People are saying that medical cannabis is just a scam and a cover for recreational use," says Ware. "I worry that the message that there 
may be medical value gets lost."

This also gives free rein for cannabis cowboys to make outlandish claims. Abrams is no longer able to keep up with the e-mails and calls about 'miracle cancer cures'. "I'm a little frustrated," he says. "There's just no data to support this." Other companies offer products that piggyback on promising preliminary research - but with little clinical proof, regulatory oversight or quality control. Hazekamp cites a recent FDA investigation of extracts from CBD-rich cannabis strains, which are being touted as a potential treatment for severe epilepsy, cancer and diabetes. "Almost none of the products complied with their labelling," he says, "and some contained no CBD at all."

\section{HIGH TIME FOR RESPONSIBLE RESEARCH}

These legal experiments could yet bear scientific fruit. Colorado is using money raised from its recreational marijuana industry to support nine clinical research grants, several of which are for observational studies of real-world patient use. In Canada, Ware helped establish the Quebec Cannabis Registry earlier this year, which will track patient health and outcomes over four years. Fischer and colleagues are developing a similar programme to monitor patients in Ontario.

There is a risk, however, that these efforts could be confounded by recreational users gaming the system. Hazekamp notes that in the Netherlands, where recreational cannabis is widely available, the proportion of people accessing medical marijuana is less than onetenth that of some US states ${ }^{6}$. Canada's medical use is also booming, and Fischer likens the situation to Prohibition in the 1920s and early 1930s, when alcohol consumption in the United States was restricted to religious and medicinal uses. "Suddenly thousands of people needed medical alcohol - and it's not like there was a sudden epidemic of ill people," he says. Observational data are also no substitute for randomized controlled trials - and ironically, medical marijuana access may impede recruitment for the trials needed to prove its value. "Those who are very ill can get cannabis anyway, and don't need a study," says Naftali.

Veteran cannabis researchers are finding new opportunities to strengthen their case. Wilsey's team is embarking on a neuropathic back-pain trial spanning 8 weeks, with more than 100 patients, while Abrams is working with NIDA's new cannabis strains to assess the combined effects of THC and CBD in modulating pain in sickle-cell anaemia - a condition associated with especially debilitating and difficult-to-manage pain. Naftali is conducting a 50-patient Crohn's disease trial that will closely examine physiological markers as well as patient-reported symptoms. "We'll be doing endoscopy before and after patients take cannabis, to see whether there's any real difference in the inflammation," she says.

Funding opportunities are also attracting

\section{CALCULATING THE COSTS}

\section{How risky is weed?}

One of the big fears of creeping legalization of marijuana is that rates of use and addiction will soar; it is estimated that nearly $9 \%$ of users become dependent ${ }^{7}$. "It's not the worst drug-use disorder, but it's also not a good thing - and the relapse rates are very high," says Margaret Haney, a neurobiologist at Columbia University in New York. She notes that dependence is particularly problematic among younger users, who are less motivated to quit than older people with families and careers.

Some evidence links heavy adolescent use to lasting impairments in cognitive development. Pharmacologist Harold Kalant at the University of Toronto, Canada, cites a 38-year-long study from New Zealand ${ }^{8}$, showing that adolescents who use the drug experience potentially lifelong deficits in cognitive skills, including judgement and working memory. "These kids have much poorer school records, higher dropout rates and poorer employment prospects," says Kalant. This was the first of only a few studies to investigate this relationship over time, so the results are open to interpretation. "It's biologically plausible," says Haney. "But there are no data that definitively demonstrate that marijuana is causing this effect." Indeed, a study published in August found no difference in mental and physical health among young adults who had used cannabis as adolescents ${ }^{9}$. However, the study was smaller and shorter than the New Zealand one and, although Haney says it was

a new generation of researchers. Colorado's research grant programme supports a placebocontrolled trial for PTSD in 76 veterans and a pain study that pits cannabis against oxycodone. Cannabis growers are also keen to build their scientific case - Canadian company National Green Biomed, based in Vancouver, gave a Can $\$ 1$ million (US\$760,000) grant to researchers at the University of British Columbia to explore whether medical marijuana suppresses HIV infection. And Bedrocan has hired its first clinical trial coordinator, and is investigating the chemical composition of its plants in order to learn why patients might claim different benefits from different strains.

The future of medical marijuana remains unclear - the money now pouring into this new industry could accelerate deregulation, or a backlash may lead to stricter controls. From Ware's perspective, the current situation may offer a fleeting chance for the research community to seize the reins (see page S9). "There's an opportunity for us to take a global leadership position - the world is crying out for some well conducted, she adds that its size makes it hard to identify real differences between users and non-users. "Those arguing marijuana might cause psychosis would probably not be dissuaded by such small numbers," she says.

One recent analysis showed that although adolescent use is higher in states that allow medical marijuana, passage of those laws did not lead to increased use suggesting that pre-existing local attitudes may be the primary factor ${ }^{10}$. Benedikt Fischer, a public health researcher at the Centre for Addiction and Mental Health in Toronto, sees the fear that cannabis is a danger to young people as an exaggerated product of 'Reefer Madness'-style moral panic. "There are a lot of hockey-related brain injuries," he says, "but nobody in this country would think about prohibiting hockey for young people." He believes that other risks, such as increased frequency of cannabis-related traffic accidents, may be under-appreciated.

Other critics see a double-standard for cannabis, given the extensive misuse of pharmaceuticals such as opioid painkillers. "There are risks, but those can be modulated by careful patient screening and public health messaging," says pain specialist Mark Ware, at McGill University in Montreal, Canada, "and they should not stop progress in considering these drugs for middle-aged women with multiple sclerosis, or for men with HIV/AIDS." M.E.

direction in managing not just medical, but also recreational cannabis," he says. "This should stop being an issue for legal minds to wrestle over; it should be the scientists that are putting the evidence together that drive the policy." -

Michael Eisenstein is a freelance science writer based in Philadelphia, Pennsylvania.

1. Abrams, D. I. et al. Neurology 68, 515-521 (2007).

2. Ware, M. A. et al. Can. Med. Assoc. J. 182, E694E701 (2010).

3. Naftali, T. et al. Clin. Gastroenterol. Hepatol. 11, 1276-1280 (2013)

4. Press, C. A., Knupp, K. G. \& Chapman, K. E. Epilepsy Behav. 45, 49-52 (2015).

5. Whiting, P. F. et al. J. Am. Med. Assoc. 313, 24562473 (2015).

6. Hazekamp, A. \& Heerdink, E. R. Eur. J. Clin. Pharmacol. 69, 1575-1580 (2013)

7. Volkow, N. D. et al. N. Engl. J. Med. 370, 2219-2227 (2014).

8. Meier, M. H. et al. Proc. Natl Acad. Sci. USA 109, E2657-E2664 (2012).

9. Bechtold, J Simpson, T. White H. R \& Pardini, D. Psychol. Addict. Behav. http://dx.doi.org/10.1037/ adb0000103 (2015).

10. Hasin, D. S. et al. Lancet Psychiatry 2, 601-608 (2015). 